\title{
Integrated Nonlinear Photonics Using CROWs (Project Report 0642603-Y1)
}

\section{SHAYAN MOOKHERJEA}

Department of Electrical and Computer Engineering, University of California, San Diego, MC 0407 La Jolla CA 92093-0407 USA

Email:smookher@ucsd.edu

\section{Introduction and Goals}

This NSF-funded project [0642603] is a five-year (60 months) CAREER (Faculty Early Career Development Program) unified research and education development program. The full title of this project is "CAREER: Chip-scale low-power nonlinear optics using coupled resonators and CROWs". The focus of this project is a study of the waveguiding principles and applications of a novel type of integrated photonics waveguide, the Coupled Resonator Optical Waveguide (CROW), in nonlinear optics at the milliwatt-scale power level, which is relevant for optical communications and signal processing.

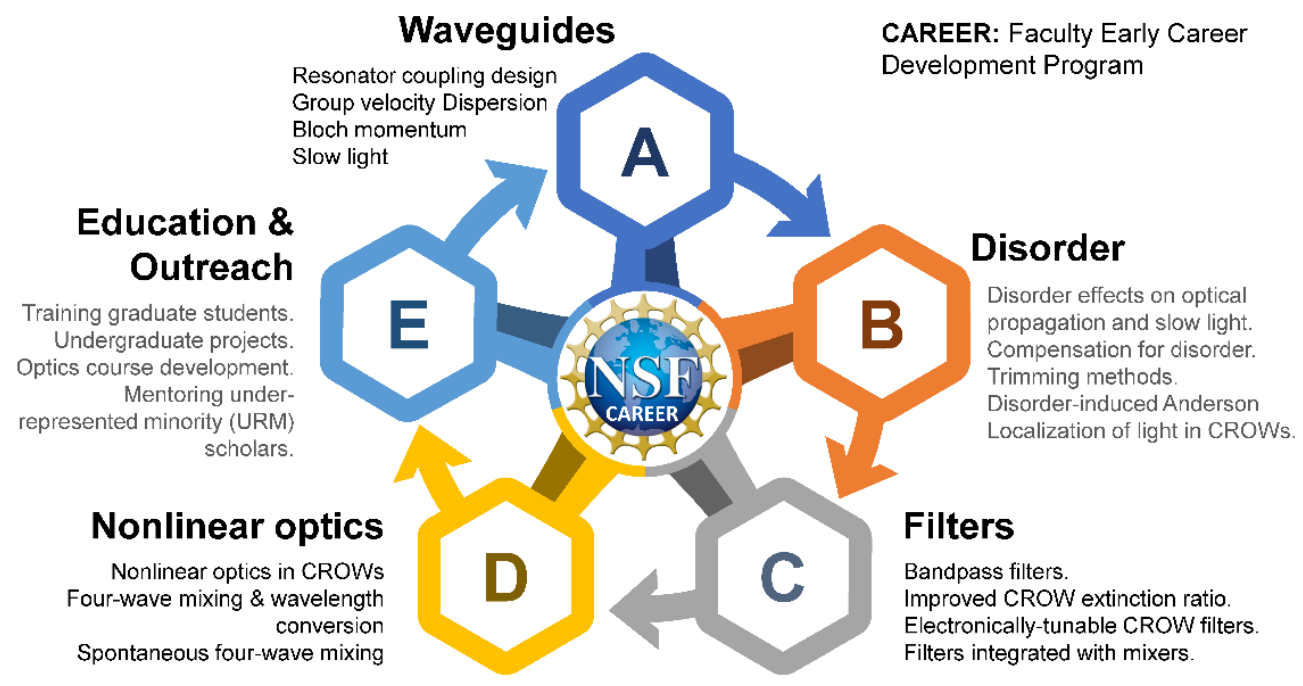

Figure 1 Project Tasks and Goals for NSF 0642603: CAREER: Chip-scale low-power nonlinear optics using coupled resonators and CROWs. (Powerpoint Template: presentationgo.com; NSF logo: NSF)

Silicon photonics is widely used for integrated photonics in optical communications. The main ultrafast nonlinear optical phenomenon which is relevant at milliwatt power levels is a third-order optical nonlinearity known as four-wave mixing (FWM). This nonlinear effect in nanoscale waveguides is efficient, and can cause an intensity-dependent phase shift, such as self-phase modulation or cross-phase modulation which is easily measurable, and is important for many other topics in nonlinear optics as well, such as soliton formation [1]-[4]. In FWM, one or more pump beams and signal beams can be 
made to interact in a silicon waveguide or, more conventionally, a microresonator to generate a new frequency, called the idler. Typically, the signal beams are modulated with high-speed digital data, resulting in wavelength-conversion of the data to the idler channel. FWM is a parametric nonlinear optical process which has been widely studied in other types of materials and waveguide structures, for example, in optical fiber [5]. The CROW structure, like other coupled-resonator structures (such as the SCISSOR [2]), is suitable as the foundation of this project because it offers a very high FWM nonlinearity based on the triple resonance of the pump, signal and idler modes in multiple passbands, and a variety of ways to achieve phase matching in real devices.

A simple example can provide a sense for the scale of the potential improvements in wavelength conversion. For a typical silicon single-mode waveguide at $1550 \mathrm{~nm}$, the four-wave mixing nonlinear coefficient is $\gamma=200$ $\mathrm{W}^{-1} \mathrm{~m}^{-1}$. In comparison, a 35-ring CROW was measured to have a slow-light enhanced value $\gamma_{\text {eff }}=4150 \mathrm{~W}^{-1} \mathrm{~m}^{-1}$ in the same wavelength regime. Since the idler power that is generated by a phase-matched FWM interaction is quadratically proportional to $\gamma$, we can expect up to $34 \mathrm{~dB}$ improvement (more than a factor of 2,000) in wavelength conversion compared to a conventional waveguide at the same power level and optical propagation length. However, there are some important issues which must be addressed in order to experimentally realize such a large improvement, include loss minimization and disorder compensation.

\section{Activities}

The first set of research activities in this project, labeled Task Area A (Waveguides) in Fig. 1, focuses on improvements and refinements to models of waveguiding in CROWs. Previously, a tight-binding model of band-center propagation was formulated [6], [7], and theoretical studies of nonlinear propagation were developed on this basis [8], [9]. However, it was predicted that propagation in the regions of high dispersion and high nonlinearity would be sensitive to disorder [10]. Newer models were developed in this project and measurements were compared to the theoretical models [11]. Thus, Task Areas A (Waveguides) and B (Disorder) are intricately linked.

Although the CROW device can have a high FWM nonlinear coefficient, optical propagation loss can also be enhanced due to slow-light effects. Disorder can cause deviations from phase matching which are also more important in the slow-light regime. Interesting physics phenomena such as localization of light can perhaps be more easily observed using the CROW structure as a testbed. Studying these effects is the focus of Task Area B.

Task Area $\mathrm{C}$ labeled in Fig. 1 addresses the design of optical filters for communications and signal processing based on the CROW structure. 
Principles of the design of optical filters and delay lines using planar lightwave circuits are already known [12], [13]. However, long CROW structures (beyond about a dozen unit cells) have not yet been realized. We focused our research on studying very high order CROWs (more than two hundred unit cells in some cases) and understanding their properties as bandpass filters and delay lines.

Studies undertaken in Task Area "D" comprise the core of this project, and lay the foundation for future device applications of CROW waveguides in nonlinear optics.

Several educational and outreach initiatives (Task Area "E") are undertaken during this NSF-funded project. An undergraduate opto-electronics course was revised with the introduction of new teaching material and new experiments. Graduate students served as mentors for high-school students from the Preuss School in San Diego, which serves an under-represented minority (URM) population. An URM post-doctoral researcher was recruited and contributed to the research activities and helped train graduate and undergraduate students.

\section{Progress and Impact}

At the time of proposal development and submission, the initial project concept was based on photonic crystal CROWs, in which slow-light propagation by "hopping" between well-confined defect resonators had been studied by several research groups [14]. As our project progressed, we focused on devices based on coupled micropillar resonators [15] and then coupled microring resonators, in which the coupling between adjacent resonators is implemented using directional couplers. The coupled-microring CROW could be fabricated more easily using conventional (photo-) lithography rather than electron-beam lithography. Silicon microring CROWs, which were fabricated on $200 \mathrm{~mm}$ SOI wafers in collaboration with IBM (Yorktown Heights) under a collaborative project (1028553) also supported by the NSF. Longer CROW structures could be made with less disorder, and in subsequent fabrication performed at IME (Singapore) also on $200 \mathrm{~mm}$ SOI wafers, electronic dopants (i.e., p-i-n junctions) were incorporated with the optical structures. (Note that the group studying photonic crystal slow light also proceeded along a similar research trajectory for their structures at about the same time [16].) Increased device length and electronic control over wave propagation were helpful in accomplishing some of the major outcomes of this project which advanced the state-of-the-art.

We experimentally studied the dispersion of such couplers using a microring coupled to a waveguide [17]. An accurate knowledge of dispersion is also necessary for coupled-microring filters [18] or tunable dispersion compensation devices [19]. To help in designing structures in silicon photonics, we developed an extension of coupled-mode theory which is applicable to directional couplers 
in high index contrast materials, in coupled-waveguide structures [20] and coupled-resonator structures [21].

Waveguiding in a CROW using microring resonators in the racetrack configuration depends upon the device geometry parameters, such as the axial periodicity (distance between adjacent microrings) $\Lambda=13.8 \mu \mathrm{m}$, effective racetrack radius $\mathrm{R}_{\text {eff }}=\mathrm{R}+\mathrm{L}_{\mathrm{c}} / \pi$ (where $\mathrm{R}=6.5 \mu \mathrm{m}$ is the arc bending radius and $\mathrm{L}_{\mathrm{c}}=20 \mu \mathrm{m}$ is the length of the directional waveguide coupler between adjacent microrings) and a geometric parameter $\Gamma=\pi R_{\text {eff }} / \Lambda=2.9$. For each passband, its band-center slowing factor $\mathrm{S}$ (ratio of the group index of the CROW to that of a conventional nanowire waveguide) is inversely proportional to the magnitude of the inter-resonator coupling coefficient $|\kappa|, S=\Gamma /|\kappa|$. The coupling coefficient can be estimated from the passband width and its value varies with wavelength. For a typical device used in our later experiments, values ranged from $|\kappa|^{2}=0.33$ near $1530 \mathrm{~nm}$ to $|\kappa|^{2}=0.49$ near $1600 \mathrm{~nm}$. The corresponding values of band-center S varied from 5.14 at $1523 \mathrm{~nm}$ to 4.1 at $1607 \mathrm{~nm}$. Studies performed using this project showed that the band-edge values of S are typically a factor of 2-3 higher.

The greater-than-unity slowing factors for each of the pump, signal and idler waves results in a large enhancement of the FWM conversion efficiency. For a representative FWM measurement that was performed towards the final years of this project, we estimated $\mathrm{S}_{\mathrm{p}}=12.1, \mathrm{~S}_{\mathrm{s}}=12.3$ and $\mathrm{S}_{\mathrm{i}}=11.1$. Thus, the effective four-wave mixing nonlinearity is:

$\gamma_{\mathrm{eff}}=\gamma_{0} \frac{s_{i} s_{i}+S_{i} \Gamma}{2 \Gamma} \sqrt{\frac{S_{s}+\Gamma}{s_{i}+\Gamma}}=4150 \mathrm{~W}^{-1} \mathrm{~m}^{-1}$.

Compared to photonic crystals, which are also slow-wave waveguides, microring CROWs have some advantages:

(i) A single coupled-microring CROW waveguide can readily achieve multiple bands of slow and filtered light in the same physical structure, unlike band-edge photonic crystal slow light waveguides.

(ii) Microring-based CROWs use the same waveguide cross-section as in conventional single-mode nanowire waveguides, which have reached a high level of maturity. Thus, they are potentially simple to integrate with other on-chip or off-chip lightwave circuitry.

On the other hand, photonic crystal CROWs are more compact devices, and the $\mathrm{Q} / \mathrm{V}$ factor (quality factor divided by the optical mode volume) is higher, which can lead to stronger light-matter interactions.

During the first year of this project, we have carried out a detailed and thorough investigation of the issue of long-range slow light propagation. This topic directly relates to the issue of disorder, arising from fabrication imperfections 
or stochastic variations in the inter-resonator coupling coefficients, on the performance of coupled resonators and the coupled-resonator waveguide. Detailed simulations have been carried out using our workstations as well as those offered by the San Diego Supercomputer Center. For the latter, we had to write our own parallelized Fortran 90 programs using MPI for the multiprocessor capabilities of the TeraGrid supercomputer.

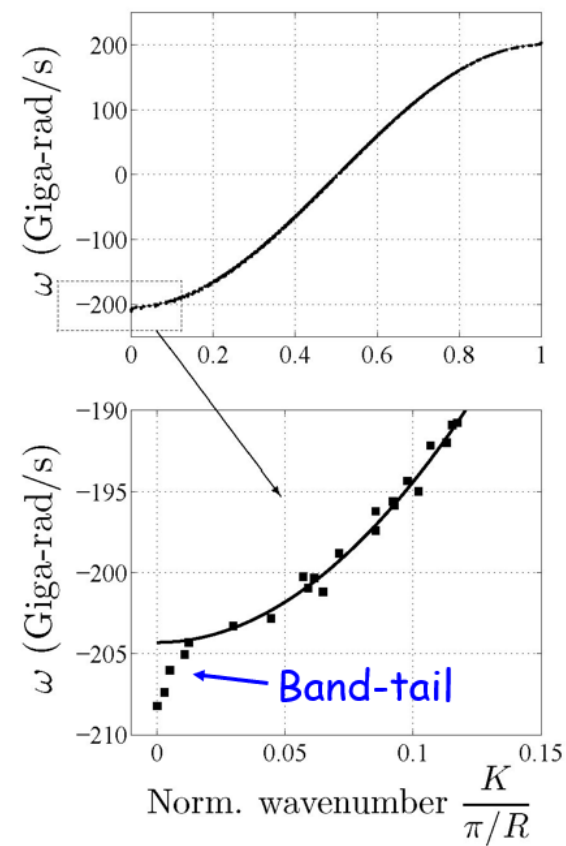

Figure 2 Calculations of the effective dispersion relationship in the weakly-disordered regime, which modifies the slow-light properties near the band edge.
We studied the tolerances required to enable long-range slow-light propagation on coupled-resonator optical waveguide. We have developed a new theoretical model for disordered slow-wave structures based on a spectral-domain representation of the system without dividing the structure into periodically-coupled unit cells. This is an alternative approach to traditional CROW calculation methods which employ the repeated unit-cell approach. Those approaches are generally unable to make an accurate prediction of the band-edge effects of disorder, which are most important for a slow-wave structure, as that is the region of greatest slowing, and phase tunability. Our calculations are able to estimate the spectral density of states $\rho(\omega)$ in the presence of disorder.

The band-center slow-light velocity is relatively insensitive to the effects of

disorder and can be studied as follows. Using $\rho(\omega) \delta \omega=\rho(\mathrm{k}) \mathrm{dk}$, we can write $v_{g}=\rho(\omega) / \rho(k)$ which can be easily calculated from the simulation. At the band center, $\rho(\omega)$ takes its minimum value. Using $\mathrm{R}=10 \mu \mathrm{m}$ as an example for coupled microrings, we estimated $\mathrm{Vg}^{(\max )} \sim 1 \times 10^{6} \mathrm{~m} / \mathrm{s}$. This agrees with the value estimated from the dispersion relationship, $\mathrm{vg}^{(\max )} \sim(\delta \omega / 2) \mathrm{R}$ where $2 \delta \omega$ is the full width of the passband.

Band-edge slow-light requires a more detailed analysis. A rule-of-thumb expression, Eq. (2) was derived from first-principles for the slowing factor near the band-edge of a CROW structure based on the disorder $\delta \kappa$ in the interresonator coupling coefficient $\kappa$ (which appears in the coupled-resonator matrix model) [22]. 


$$
S=\frac{c}{v_{g(\text { band-edge })}} \approx \frac{\lambda}{R} \frac{1}{\left(\delta \kappa^{2} \kappa\right)^{\frac{1}{3}}}
$$

For variations in the inter-resonator coupling coefficients in the range of $1 \%$ to $10 \%$, which covers the range of practical interest with current fabrication technology, slow light at the band edge is expected to be only 10 to 30 times slower than at the band center. This was in good agreement with experimental CROW measurements made a few years earlier in both the optical and microwave $(\mathrm{RF})$ regimes.

Impact on Human Resources: Two graduate students in the PI's group worked on this topic ( $>160$ hours) as part of their education and training. One of the students was supported by an NSF Graduate Student Fellowship. One undergraduate student contributed to the research efforts on modeling disorder and co-authored a journal paper. The San Diego Supercomputer Center provided access to computing facilities.

\section{Subsequent Extensions}

Other than the focus on FWM, there are other nonlinear optical effects in coupled microring resonators. For example, when the optical power is increased, silicon microring resonators with directional couplers can exhibit interesting behavior, such as bistability and nonlinear on-off switching [23].

We seek a control "knob" to tune the waveguiding properties of the CROW structure. We decided to use electro-optic tunability for most of our studies. Using electro-optic control fields was practically convenient since it was easy to generate the switching waveforms in the laboratory [24]. Integrated electrooptic modulators could be another way of achieving phase shifters with more than $100 \mathrm{GHz}$ of bandwidth, as was demonstrated much later [25]. If tuning speed is not a concern, the thermo-optic effect, which we used for tunable filters, is also another option as a control knob [26].

A collaboration with IBM's silicon photonics group (Dr. W. M. J. Green) provided insights into silicon photonics fabrication and also supported technical collaboration on fabricating the CROW structures [11], [27], [28]. Dr. Ivan B. Divliansky (Research Scientist, CREOL, Florida) collaborated with us for the electron-beam lithography of long waveguide structures [29]. CROWs of up to 235 coupled resonators were successfully fabricated and measured, and a good agreement was achieved between experiments and theory [28], [30], [31].

Infrared imaging is a useful experimental tool to study light propagation through the length of these structures [29], [32]. Novel measurement techniques were developed based on a single-scan, Jones matrix-based, interferometric spectral measurement instrument (Luna Tech. Optical Vector Analyzer OVA5000, generously loaned by Luna Technologies, VA and subsequently 
used in an innovative way in our measurements [33]. A jointly-authored publication resulted from this collaboration [11].

The CROW structure can have other applications including generation of quantum light [34], and developing amplifiers and lasers with novel properties [35]. We developed a model for spontaneous four-wave mixing (SFWM) using the Collett-Gardiner approach [34]. This method formulates the time-domain equations of motion in the Heisenberg picture. We solved the equations in the case of a single resonator as well as for multiple resonators, showing the potential advantages of CROWs. Our initial studies of spontaneous four-wave mixing (SFWM) were on correlated photon-pair generation and heralded single-photon generation [36]. An experimental measurement of entanglement was performed later, using a two-photon interferometer constructed to verify time-energy entanglement [37].

Microring resonators are sensitive to disorder. If nanoscale disorder can be modified, device performance may be improved. We studied potential methods for precisely tuning resonators without heaters. Our method is based on fieldinduced local oxidation of $\mathrm{Si}$ to $\mathrm{SiO}_{2}$ via a chemical reaction near an electrically-biased conducting atomic-force microscope tip [38], [39]. Scanning probe lithography has previously been used to modify the resonance frequency of a GaAs photonic crystal cavity, and is part of a larger class of methods developed for physical and chemical surface modification. A single silicon microresonator can be monitored and controlled more easily [40] than a long CROW device.

\section{Open-Access Reporting Initiative}

PRAISE: This open-access document is provided in support of our PRAISE

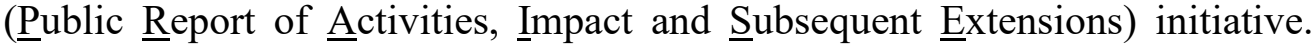
What is it? An open-access document shared with the public which describes the research outcomes of publicly-funded projects. For us, these projects are typically funded by the NSF (National Science Foundation).

\section{References}

[1] J. E. Heebner, R. W. Boyd, and Q. H. Park, "Slow light, induced dispersion, enhanced nonlinearity, and optical solitons in a resonator-array waveguide," Phys. Rev. E, vol. 65, no. 3, p. 036619, 2002.

[2] J. E. Heebner, R. W. Boyd, and Q.-H. Park, "SCISSOR solitons and other novel propagation effects in microresonator-modified waveguides," $J$. Opt. Soc. Am. B, vol. 19, pp. 722-731, 2004.

[3] A. Ciattoni, B. Crosignani, S. Mookherjea, and A. Yariv, "Nonparaxial dark solitons in optical Kerr media," Opt. Lett., vol. 30, no. 5, p. 516, Mar. 2005, doi: 10.1364/OL.30.000516. 
[4] B. Crosignani, A. Yariv, and S. Mookherjea, "Nonparaxial spatial solitons and propagation-invariant pattern solutions in optical Kerr media," Opt. Lett., vol. 29, no. 11, p. 1254, Jun. 2004, doi: 10.1364/OL.29.001254.

[5] R. Stolen, "Phase-matched-stimulated four-photon mixing in silica-fiber waveguides," IEEE J. Quantum Electron., vol. 11, no. 3, pp. 100-103, Mar. 1975, doi: 10.1109/JQE.1975.1068571.

[6] S. Mookherjea and A. Yariv, "Optical pulse propagation in the tightbinding approximation," Opt. Express, vol. 9, no. 2, p. 91, Jul. 2001, doi: 10.1364/OE.9.000091.

[7] S. Mookherjea and A. Yariv, "Pulse propagation in a coupled resonator optical waveguide to all orders of dispersion," Phys. Rev. E, vol. 65, no. 5, p. 056601, Apr. 2002, doi: 10.1103/PhysRevE.65.056601.

[8] S. Mookherjea and A. Yariv, "Kerr-stabilized super-resonant modes in coupled-resonator optical waveguides," Phys. Rev. E, vol. 66, no. 4, p. 046610, Oct. 2002, doi: 10.1103/PhysRevE.66.046610.

[9] S. Mookherjea and A. Yariv, "Optical pulse propagation and holographic storage in a coupled-resonator optical waveguide," Phys. Rev. E, vol. 64, no. 6, p. 066602, Nov. 2001, doi: 10.1103/PhysRevE.64.066602.

[10] S. Mookherjea, D. S. Cohen, and A. Yariv, "Nonlinear dispersion in a coupled-resonator optical waveguide," Opt. Lett., vol. 27, no. 11, p. 933, Jun. 2002, doi: 10.1364/OL.27.000933.

[11] M. L. Cooper et al., "Waveguide dispersion effects in silicon-oninsulator coupled-resonator optical waveguides," Opt. Lett., vol. 35, no. 18, p. 3030, Sep. 2010, doi: 10.1364/OL.35.003030.

[12] C. K. Madsen and J. H. Zhao, Optical Filter Design and Analysis. New York: John Wiley \& Sons, 1999.

[13] G. Lenz, B. J. Eggleton, C. K. Madsen, and R. E. Slusher, "Optical delay lines based on optical filters," IEEE J. Quantum Electron., vol. 37, no. 4, pp. 525-532, Apr. 2001, doi: 10.1109/3.914401.

[14] T. Baba, "Slow light in photonic crystals," Nat. Photonics, vol. 2, no. 8, pp. 465-473, 2008.

[15] S. Mookherjea, J. S. Park, S.-H. Yang, and P. R. Bandaru, "Localization in silicon nanophotonic slow-light waveguides," Nature Photon, vol. 2, no. 2, pp. 90-93, Feb. 2008, doi: 10.1038/nphoton.2007.278.

[16] R. Hayakawa, N. Ishikura, H. C. Nguyen, and T. Baba, "High-speed delay tuning of slow light in pin-diode-incorporated photonic crystal waveguide," Opt. Lett., vol. 38, no. 15, p. 2680, Aug. 2013, doi: 10.1364/OL.38.002680.

[17] R. Aguinaldo, Yiran Shen, and S. Mookherjea, "Large Dispersion of Silicon Directional Couplers Obtained via Wideband Microring Parametric Characterization," IEEE Photon. Technol. Lett., vol. 24, no. 14, pp. 1242-1244, Jul. 2012, doi: 10.1109/LPT.2012.2198639. 
[18] J. R. Ong, R. Kumar, and S. Mookherjea, "Silicon microring-based wavelength converter with integrated pump and signal suppression," Opt. Lett., vol. 39, no. 15, p. 4439, Aug. 2014, doi: 10.1364/OL.39.004439.

[19] S. Mookherjea, "Using gain to tune the dispersion relation of coupledresonator optical waveguides," IEEE Photon. Technol. Lett., vol. 18, no. 5, pp. 715-717, Mar. 2006, doi: 10.1109/LPT.2006.871144.

[20] M. L. Cooper and S. Mookherjea, "Numerically-assisted coupledmode theory for silicon waveguide couplers and arrayed waveguides," Opt. Express, vol. 17, no. 3, p. 1583, Feb. 2009, doi: 10.1364/OE.17.001583.

[21] S. Mookherjea, "Spectral characteristics of coupled resonators," $J$. Opt. Soc. Am. B, vol. 23, no. 6, p. 1137, Jun. 2006, doi: 10.1364/JOSAB.23.001137.

[22] S. Mookherjea and A. Oh, "Effect of disorder on slow light velocity in optical slow-wave structures," Opt. Lett., vol. 32, no. 3, p. 289, Feb. 2007, doi: $10.1364 /$ OL.32.000289.

[23] S. Mookherjea and M. A. Schneider, "The nonlinear microring adddrop filter," Opt. Express, vol. 16, no. 19, p. 15130, Sep. 2008, doi: 10.1364/OE.16.015130.

[24] S. Mookherjea, J. R. Ong, X. Luo, and L. Guo-Qiang, "Electronic control of optical Anderson localization modes," Nature Nanotech, vol. 9, no. 5, pp. 365-371, May 2014, doi: 10.1038/nnano.2014.53.

[25] X. Wang, P. O. Weigel, J. Zhao, M. Ruesing, and S. Mookherjea, "Achieving beyond-100-GHz large-signal modulation bandwidth in hybrid silicon photonics Mach Zehnder modulators using thin film lithium niobate," APL Photonics, vol. 4, no. 9, p. 096101, Sep. 2019, doi: 10.1063/1.5115243.

[26] R. Aguinaldo et al., "Wideband silicon-photonic thermo-optic switch in a wavelength-division multiplexed ring network," Opt. Express, vol. 22, no. 7, p. 8205, Apr. 2014, doi: 10.1364/OE.22.008205.

[27] J. R. Ong et al., "Low-power continuous-wave four-wave mixing in silicon coupled-resonator optical waveguides," Opt. Lett., vol. 36, no. 15, pp. 2964-2966, 2011.

[28] M. L. Cooper et al., "235-ring Coupled-Resonator Optical Waveguides," in Conference on Lasers and Electro-Optics 2010, San Jose, California, 2010, p. CTuHH3. doi: 10.1364/CLEO.2010.CTuHH3.

[29] M. L. Cooper, G. Gupta, J. S. Park, M. A. Schneider, I. B. Divliansky, and S. Mookherjea, "Quantitative infrared imaging of silicon-on-insulator microring resonators," Opt. Lett., vol. 35, no. 5, p. 784, Mar. 2010, doi: 10.1364/OL.35.000784.

[30] S. Mookherjea and M. A. Schneider, "Avoiding bandwidth collapse in long chains of coupled optical microresonators," Opt. Lett., vol. 36, no. 23, p. 4557, Dec. 2011, doi: 10.1364/OL.36.004557. 
[31] M. L. Cooper and S. Mookherjea, "Modeling of Multiband Transmission in Long Silicon Coupled-Resonator Optical Waveguides," IEEE Photon. Technol. Lett., vol. 23, no. 13, pp. 872-874, Jul. 2011, doi: 10.1109/LPT.2011.2141657.

[32] S. Mookherjea and H. R. Grant, "High dynamic range microscope infrared imaging of silicon nanophotonic devices," Opt. Lett., vol. 37, no. 22, p. 4705, Nov. 2012, doi: 10.1364/OL.37.004705.

[33] M. L. Cooper, M. A. Schneider, G. Gupta, and S. Mookherjea, "FilterLess Amplification for Rapid Swept-Wavelength Interferometric Measurement of Silicon Waveguide Group Delay Statistics," IEEE Photon. Technol. Lett., vol. 23, no. 12, pp. 783-785, Jun. 2011, doi: 10.1109/LPT.2011.2134842.

[34] J. R. Ong and S. Mookherjea, "Quantum light generation on a silicon chip using waveguides and resonators," Opt. Express, vol. 21, no. 4, p. 5171, Feb. 2013, doi: 10.1364/OE.21.005171.

[35] S. Mookherjea, "Semiconductor coupled-resonator optical waveguide laser," Appl. Phys. Lett., vol. 84, no. 17, pp. 3265-3267, Apr. 2004, doi: 10.1063/1.1719278.

[36] M. Davanço et al., "Telecommunications-band heralded single photons from a silicon nanophotonic chip," Appl. Phys. Lett., vol. 100, no. 26, p. 261104, Jun. 2012, doi: 10.1063/1.4711253.

[37] R. Kumar, M. Savanier, J. R. Ong, and S. Mookherjea, "Entanglement measurement of a coupled silicon microring photon pair source," Opt.

Express, vol. 23, no. 15, p. 19318, Jul. 2015, doi: 10.1364/OE.23.019318.

[38] Y. Shen, I. B. Divliansky, D. N. Basov, and S. Mookherjea, "Electricfield-driven nano-oxidation trimming of silicon microrings and interferometers," Opt. Lett., vol. 36, no. 14, p. 2668, Jul. 2011, doi: 10.1364/OL.36.002668.

[39] Y. Shen, I. B. Divliansky, D. N. Basov, and S. Mookherjea, "Perfect set-and-forget alignment of silicon photonic resonators and interferometers," in Optical Fiber Communication Conference/National Fiber Optic Engineers Conference 2011, Los Angeles, California, 2011, p. PDPC3. doi: 10.1364/OFC.2011.PDPC3.

[40] M. Savanier, R. Kumar, and S. Mookherjea, "Optimizing photon-pair generation electronically using a $p-i-n$ diode incorporated in a silicon microring resonator," Appl. Phys. Lett., vol. 107, no. 13, p. 131101, Sep. 2015, doi: 10.1063/1.4932047. 